\title{
Use of DNA Methylation for Cancer Detection and Molecular Classification
}

\author{
Jingde $\mathrm{Zhu}^{1,2,3, *}$ and Xuebiao $\mathrm{Yao}^{4,5}$ \\ ${ }^{1}$ Cancer Genetics and Gene Therapy program, The State-key Laboratory for Oncogenes and Related Genes, Shanghai Cancer Institute, \\ Shanghai Jiaotong University and ${ }^{2}$ Epigenomics program, Shanghai Center for Systems Biomedicine, \\ Shanghai Jiao Tong University, LN 25/2200, Xie-tu Road, Shanghai 200032, China \\ ${ }^{3}$ School of Life Sciences, Zhejiang Science and Technology University, Xiasha University District, Hangzhou, Zhejiang 310018, China \\ ${ }^{4}$ Division of Cellular Dynamics, Hefei National Laboratory, Hefei 230027, China \\ ${ }^{5}$ Cancer Biology Program, Morehouse School of Medicine, 720 Westview Drive, Atlanta, GA 30310, USA
}

Received 20 February 2007

\begin{abstract}
Conjugation of the methyl group at the fifth carbon of cytosines within the palindromic dinucleotide 5'-CpG-3' sequence (DNA methylation) is the best studied epigenetic mechanism, which acts together with other epigenetic entities: histone modification, chromatin remodeling and microRNAs to shape the chromatin structure of DNA according to its functional state. The cancer genome is frequently characterized by hypermethylation of specific genes concurrently with an overall decrease in the level of 5-methyl cytosine, the pathological implication of which to the cancerous state has been well established. While the latest genome-wide technologies have been applied to classify and interpret the epigenetic layer of gene regulation in the physiological and disease states, the epigenetic testing has also been seriously explored in clinical practice for early detection, refining tumor staging and predicting disease recurrence. This critique reviews the latest research findings on the use of DNA methylation in cancer diagnosis, prognosis and staging/classification.
\end{abstract}

Keywords: Cancer DNA Methylation, Epigenetics, Methylation Specific PCR

\section{Introduction}

Having the genetic blueprints for human and the increasing number of model organisms available has ushered in a new era for the functional elucidation of genetic makeup in both development and diseases. It chiefly concerns the epigenetic layer of genetic information; that is, inheritable component

\footnotetext{
*To whom correspondence should be addressed.

Tel: 86-21-64224285; Fax: 86-21-64224285

E-mail: zhujingde@yahoo.com
}

without changes in DNA sequence which consists of four definable entities, i.e., DNA methylation (cytosine [CpG] methylation), non-coding RNA, histone modification, and chromatin remodeling. The epigenetic interface sits between the genetic blueprints stored in DNA sequences and phenotypes dictated by the pattern of gene expression and more readily responds to the changing environment than its genetic counterparts with the profound biological consequences. Addition of the methyl group at cytosine ring within 5'-CpG3' sequence (Fig. 1) is carried out by one of three DNA methyl transferase genes (DNMT1, DNMT3a, and DNMT3b) with Sadenosyl methionine as the methyl donor (Bird,1992). The DNA methylation pattern in the parental cells can be faithfully duplicated and distributed into daughter cells in a fashion similar to the semi-conservative mechanism for precise replication of the genetic information encoded in DNA sequence. For the lasting transcription memory, DNA methylation is the preferred mechanism, regardless of the nature of the first epigenetic hit responsible for the changed transcription state. Between the major epigenetic reprogramming taken place in the early embryonic development and germ cell maturation, the epigenetic make-up is also moderately altered throughout the life of living organisms (Jaenisch and Bird, 2003). A integrated epigenetic homeostatic mechanism is required to monitor and repair any epigenetic abnormalities, failure of which leads to an accumulation of the epigenetic lesions beyond repair and ultimately the various diseases states, including cancer (Baylin and Ohm, 2006; Ting et al., 2006).

\section{Cancer is a disease of aberrant DNA methylation}

Cancers are extremely complex diseases with distinct combinations of genetic and epigenetic defects that vary with both types of diseases and individuals (Hanahan and Weinberg, 2000), thus challenging the current biomedical and 
molecular knowledge and technology. Epigenetic mechanisms comprise of DNA methylation, histone modification, microRNAs and chromatin remolding that work together to control the chromatin structure of DNA to meet the functional requirements. DNA methylation, an enzymatic process to add the methyl group at the fifth carbon of cytosines within the palindromic dinucleotide 5'-CpG-3' sequence (DNA methylation) (Fig. 1) is the best studied epigenetic mechanism and the focal point of the present cancer epigenetic study for better management of these devastating diseases.

Over $85 \% \mathrm{CpG}$ dinucleotides are spread out in genome and located in the repetitive sequences having the transcriptiondependent transposition potential. They are heavily hypermethylated/transcription-silenced in the normal cells, a state crucial to the integrity of the chromatin structure of genome. The global genomic hypomethylation found in all types of human cancer studied is instrumental to the unwanted transcription of the otherwise methylated/silenced repetitive sequences and the proto-oncogenes (Bird, 1992; Baylin and Ohm, 2006), predisposition to the chromosome instability (Eden et al., 2003; Gaudet et al., 2003). The remaining circa $15 \% \mathrm{CpG}$ are clustered within the short DNA regions (approximately, 0.2 to $1 \mathrm{~kb}$ in length, called " $\mathrm{CpG}$ island" which accounts for $1 \%$ of the genome. Approximately $40-$ $50 \%$ of the genes have $\mathrm{CpG}$ island within or around the promoter and are largely unmethylated in the normal somatic cells, except for the silenced allele of the imprinted genes and the cell lineage-specific genes in the lineages where their expression is not required. However, the hypermethylationdependent silencing in cancer cells have been reported for the classic tumor suppressor genes, DNA repair genes, cell cycle control genes, anti-apoptotic genes, and genes that prevent abnormal activity of developmental pathways. The epigenetic regulation does not work independently from its genetic counterpart as vividly demonstrated by the fact that the hypermethylated promoter $\mathrm{CpG}$ island/transcription silencing affects exclusively the wild-type allele (Esteller et al., 2001) in colon cancer. To cope with the genotoxic effects of both external and internal origins, living organisms are equipped with a large array of mechanisms, including over 200 genes that involve in sensing and repairing the damages to protect the genome integrity. However, the expression of over $75 \%$ of the DNA repair genes are under the control by the DNA methylation mediated mechanisms (http://www.ncbi.nlm.nih.gov/entrez/ query.fcgi?CMD=search\&DB=gene). The hypermethylated/ transcription silencing state of several DNA repairing genes has been suggested seminal to cancer formation, by leaving the mutated DNA sequences un-repaired. Indeed, the G-to-A type mutation of the Ras proto-oncogene and the $p 53$ tumor suppressor gene were more frequent in the colorectal cancer tissues where the hypermethylated/transcriptionally silenced $M G M T$ gene resides, the protein of which prevents the G-to-A mutation (Esteller, 2000; Esteller et al., 2001). Furthermore, methylation sensitizes the CG-to-TG transition in DNA, which is thought to be responsible for the so-called $\mathrm{CpG}$ depletion in mammals through evolution (Bird, 1986) and the frequent TG mutation in tumor-associated genes (p53) in cancer (http://www-p53.iarc.fr/). The UV radiation mediated $\mathrm{CC}>\mathrm{TT}$ transversion takes place more frequently when $\mathrm{C}$ is methylated (Tommasi et al., 1997). Preference of the methylated to the unmethylated DNA has been reported for a number of the nucleic acid binding carcinogens (Chen et al., 1998). Finally, the epigenetic makeup, than its genetic counterpart, is more susceptible to the changing surroundings, placing it as a cause for the genetic changes in cancer, which are a direct consequence of the environmental insults by the traditional wisdom. The fact that the majority of carcinogens are non-genotoxic has further strengthened this view (Trosko and Upham, 2005).

The critical role of the epigenetic abnormality at the initiation stage of cancer formation was first suggested by the association of the loss of genetic imprinting (LOI) for the maternal source in tetartocarcinoma and the paternal source in hydatidiform moles/choriocarcinomas (Jelinic and Shaw, 2007). Indeed, the LOI of IGF2 gene, over-expression of which promotes cell proliferation, was found in normal-appearing colonic epithelium of patients with colorectal cancer and the increased risk of colon cancer has been reported for the individuals with LOI of this gene in circulating white cells (Cui et al., 2003). The hypermethylated/transcription silencing of the tumor suppressor and DNA repairing genes prevails at the pre-neoplastic stage of cancer formation (Belinsky et al., 1998; Belinsky, 2004). For instance, the hypermethylated pl6ink4A (tumor suppressor gene) and MGMT (the DNA repairing gene) were found in the sputum DNA as early as 35 months before lung cancer have been diagnosized (Palmisano et al., 2000). The genetic studies with the animal models of the germline mutations have demonstrated the role at the initiation stage in cancer formation of p16ink $4 \mathrm{~A}$ in lung cancer, which correlates with a prolonged expansion life of the stem cell population (Janzen et al., 2006) and the subsequent epigenetic silencing of other crucial genes (Reynolds et al., 2006). This is consistent with the notion that would increase the stem cell expansion with an increased chance of abnormalities (Hanahan and Weinberg, 2000). Indeed, the Wnt signaling circuit has been found overactive in colon cancer via both mutations in downstream effector genes such as APC and $\beta$-catenin (Kinzler and Vogelstein, 1996; Radtke and Clevers, 2005) and epigenetic silencing of the SFRPs, which encode for proteins that antagonize Wnt activation at the cell membrane (Suzuki et al., 2002; Suzuki et al., 2004). In favor of this notion, embryonic fibroblasts from the mice with substantial loss of gene imprinting by the regulated disruption of the maintenance DNA methyltransferase, Dnmt1 (Holm et al., 2005) formed tumors in immuno-compromised mice. The same genetically modified cells behave as the spontaneously immortalized cells in vitro and create multiple tumors in chimerical animals originated (Holm et al., 2005). Therefore, a switch in heritable gene expression patterns, in the absence of mutations, may lead to abnormal expansion of 
stem/progenitor cells and, thus increase the chance that full transformation and evolution of cancer occur. Recent studies in gastric cancer has strongly suggested for the association of the risk factors as such Helicobacter pylori infection and a unique aberrant DNA methylation pattern (Ushijima et al., 2006). Therefore, utility of the DNA methylation where serum, stool, sputum and urine sediments are used as the tumor DNA resources in population screening scheme for occult neoplasia sufferers or the high risk groups have been also seriously considered (Jubb et al., 2003).

As genetic defects, the epigenetic abnormalities can occur at all the stages of cancer progression, some of which are responsible for the altered behavior specific to given stages, such as the dysplasia, local benign and malignant tumor and finally metastatic lesions. Therefore, the information of the altered DNA methylation in the context of the cancer progression should have a great value in refining the staging and classification of cancer and provide the guidance for the better clinical management of cancers.

\section{DNA methylation for cancer detection}

The cancer associated altered $\mathrm{CpG}$ methylation can readily be detected against a background of normal DNA where the targeted $\mathrm{CpG}$ is at an opposite state of DNA methlylation to the former, by the methylation-specific PCR (MSP) (Herman et al., 1996) of the bisulphite treated DNA where the unmethylated by converting to uridine but not the methylated CpG. MSP is a sensitive assay, detecting one hypermethylated allele in the presence of 1,000-10,000 the unmethylated alleles (Herman et al., 1996). Using the quantitative version of MSP assay (MethyLight), the sensitivity reaches 1/100,000 without a loss of specificity and the need for gel electrophoresis (Eads et al., 2000). The nested PCR version of MSP could further increases the sensitivity, but at the expense of specificity (Palmisano et al., 2000). The methylation status of DNA is both chemically and biologically stable. Therefore, tumorassociated altered methylated DNA has been identified in various body fluids and/or the circulation of patients with esophageal, lung, breast, head and neck, prostate, and colonrectal cancer by MSP or quantitative MSP (Table 1).

DNA methylation offers other distinct advantages over genetic markers (Table 1). It is a positive signal, opposed to negative signals (e.g., LOH), which are masked by contamination with normal cells (Kolble et al., 1999). Secondly, an oncogene or tumor suppressor gene may be altered/inactivated by any one of many nucleotide sequences of the coding and noncoding regions of the genes. Alteration of an amino acid can be changes across three nucleotides of codon, each of which can be substituted by any one of a few nucleotides, thus necessitating several PCR reactions to identify all possible alterations responsible for a single amino acid alteration. There are over 23,544 somatic mutations, 376 germline mutations have been reported for the p53 coding region in cancer (http://www-p53.iarc.fr/). It is not possible to have all the possible variations to cover all the known mutation in $p 53$ gene analyzed even with the most powerful platform technology at the present time. Furthermore, the genetic effects within it's non-coding region has not or rarely been surveyed in cancer. On the contrary, epigenetic "defects" are almost always associated with hypermethylation within a defined promoter-region CpG island, and a single MSP PCR reaction (usually for the methylated allele) is needed.

Furthermore, genetic lesions are not specific for particular tumor tissue types. Consequently, if the circulating DNA in serum or plasma is used for screening, it is not possible to pinpoint the location of the occult tumor. On the contrary, the set of hypermethylated $\mathrm{CpG}$ islands can be specific to a given tumor type, allowing the investigator to identify the origin of the altered DNA (Table 2). In last few years, we have methylation-profiled of six types of common cancer in China to identify the informative set of genes for differentially detection of one from other cancer types (Yu et al., 2002; Yu et al., 2003; Ding et al., 2004; Gu et al., 2006; Li et al., 2004; $\mathrm{Xu}$ et al., 2004; Xu et al., 2004; Yang et al., 2004; Yu et al., 2004; Yu et al., 2004; Zhu, 2005; Zhu, 2006; Huang et al., 2006; unpublished obserrations). From no less 31 genes profiled in the cancer tissues of no less than 70 cases, no less 19 genes are informative to a given type of cancer. As listed in

Table 1. Molecular biomarkers for cancer detection

\begin{tabular}{lcccc}
\hline & Genetic & Epigenetic & \multicolumn{2}{c}{ Expressional } \\
\cline { 2 - 5 } & Mutation, SNP, LOH & DNA methylation & mRNA & Protein \\
\hline Stability & High & High & Low & Low \\
PCRable & Yes & Yes & Yes & No \\
Target/gene & Multiple & Single & NA & Quantitative \\
Nature & Quantitative & Qualitative & Quantitative & Essential \\
Sample purity & Essential & Moderate & Essential & Yes \\
Fluctuation & No & No & No/Low & No/Low \\
Tumor type specificity & No & High &
\end{tabular}

NA, not applicable; multiple, more than one target (single) needs to be analyzed; fluctuation, whether the amount of the biomarker changes according to the fluctuation of nontumor factors (emotional, physiological, or pathological factors) 
Table 2, The type specific methylation pattern (Occurrence, \%)

\begin{tabular}{lcccrrrr}
\hline Type (No. of cases) & $\begin{array}{c}\text { No. of genes } \\
\text { tested }\end{array}$ & $\begin{array}{c}\text { No. of genes } \\
\text { informative }\end{array}$ & CDH13 & MYOD1 & MGMT & p16INK4a & RASSF1A \\
\hline Bladder Cancer (209) & 66 & 28 & 3.8 & 14.3 & 0.0 & 6.3 & 11.9 \\
Colon Cancer (64) & 31 & 19 & 63.5 & 68.3 & 19.0 & 15.9 & 3.2 \\
Glioma (57) & 95 & 28 & 5.7 & 1.9 & 35.8 & 1.9 & 69.8 \\
Liver Cancer (184) & 136 & 47 & 28.9 & 27.1 & 3.6 & 34.3 & 97.0 \\
Lung Cancer (89) & 100 & 42 & 48.1 & 35.1 & 22.1 & 26.0 & 51.9 \\
Ovarian Cancer (235) & 47 & 39 & 28.7 & 10.4 & 2.3 & 7.5 & 46.6 \\
\hline
\end{tabular}

Table 2, the occurrence of the hypermethylated state of a five gene set varies significantly with the cancer type (Table 2), thus distinguishing one from the other types of cancer.

Both RNA and protein based biomarkers, underlining the phenotypic uniqueness of cancer cells have been most intensively evaluated for their utility in cancer detection for more than a decade. However, the following features prevent their use in clinical practice. Both markers are biologically and biochemically unstable, making recovery of them in bodily fluid difficult and their readout suffers from sample contamination and illegitimate expression. (reviewed in Macdonald, 1999; Tsavellas et al., 2001).

Despite of all the advantages, DNA methylation based method for cancer detection remains at its infant stage. Lack of the informative set of the promoter $\mathrm{CpG}$ islands for particular cancer types and the practical and regulatory concerns in the clinical evaluation are two major hurdles. Therefore, our efforts to establish a DNA methylated method to detect bladder cancer in urine sediments started with screening for the informative set of 58 tumor associated genes in three established bladder cancer cell lines, bladder cancer tissues and urine sediments. Then 21 genes were methylated profiled in the urine sediments of the bladder cancer (132 cases), the non-cancerous urinary lesion (23 cases), the nonurinogenitary disease (6) cases and the healthy volunteers (7) cases. Among the controls, only 3 of 23 non-cancerous urinary lesion patients were found associated with three hypermethylated genes. 121 among 132 (91.7\%) bladder cancer cases have at least one hypermethylated of 11 genes analyzed, with a decent specificity: $87 \%$ (manuscript in submission). We are currently evaluating this set of the methylated genes for bladder cancer detection in a large cohort of patients with the urinary symptoms. At the same time, searching for more informative targets by a genomewide approach involving probing the oligonucleotide $\mathrm{CpG}$ island array with the affinity purified heavy methylated DNA fragments is underway (unpublished observation).

Hepatocellular carcinoma (HCC) is one of the most aggressive malignancies (http://www-depdb.iarc.fr/globocan/ GLOBOframe.htm). Difficulties in the early diagnosis and clinical management, such as both inherent and adaptive resistance to the common chemotherapeutic modalities, and its rapidly advancing nature contribute to the poor clinical

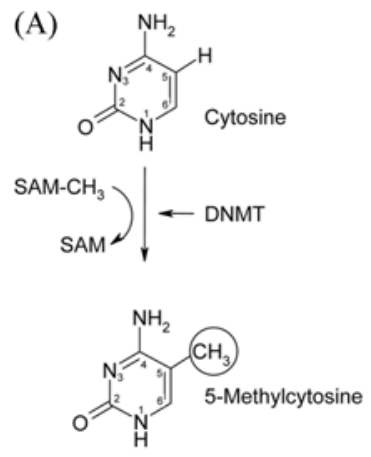

(B)

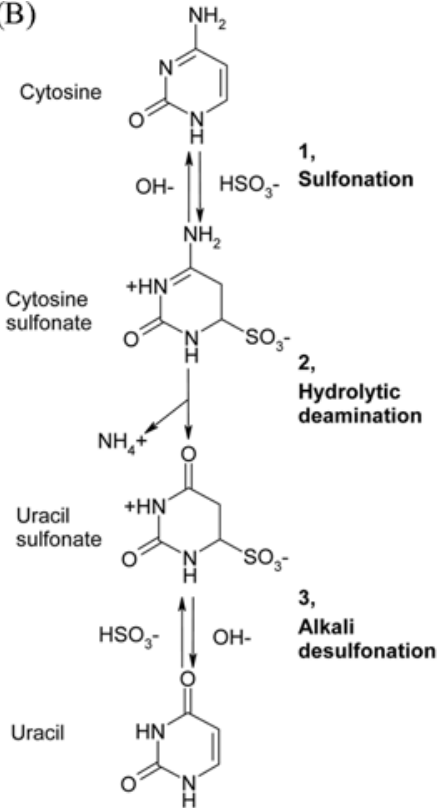

Fig. 1. Cytosine (CpG) methylation. (A) DNA methyltransferases $1,3 \mathrm{a}$, or $3 \mathrm{~b}(D N M T)$ catalyzes the addition of a methyl group (the circled $\mathrm{CH} 3$ ) at the fifth carbon of the pyrimidine ring of the cytosine nucleotide by using S-adenosyl methionine (SAMCH3) as a methyl donor. (B) C-to-T transition is initiated by the sulfonation of cytosine (1, cytosine to cytosine sulfonate), then hydrolytic deamination occurs (2, cytosine sulfonate to uracil sulfonate), with the process concluded by alkali desulfonation (3, uracil sulfonate to uracil). Methyl cytosine resists this chemical treatment; thus, methylated versus unmethylated $\mathrm{CpG}$ can be detected by a subsequent polymerase chain reaction (PCR), including methylation-specific PCR.

outcome of HCC sufferers, which is largely attributed to the inadequancy of the staging and classification system based upon clinical observations, imaging, and biochemical data (Gospodarowicz et al., 2004). In last few years, we have methylation profiled by MSP of methylation 136 promoter $\mathrm{CpG}$ island in 184 liver cancer tissues (with the pair noncancerous tissues) and 6 healthy liver tissue, found that the 47 genes, while unmethylated in the normal control, were hypermethylated to various extents in the liver cancer tissues (unpublished observations). It has been generally accepted 
(A)

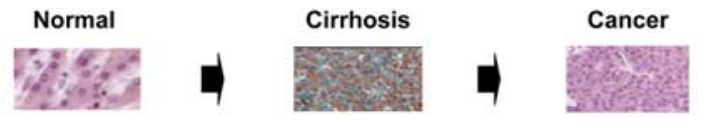

(B)

\section{The altered methylation pattern}
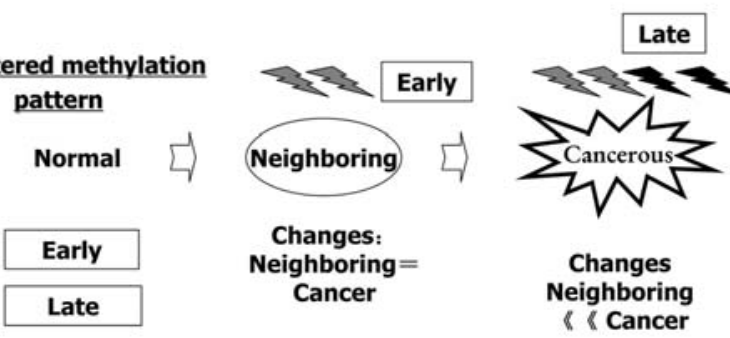

DNA methylation based staging and classification of liver cancer

Fig. 2. DNA Methylation Profile of Liver Cancer for Classification and Staging. (A) Tissue will be collected from the normal healthy liver donor, cirrhotic liver and liver cancer tissues (paired non-tumorous tissues) for methylation profiling. (B) The altered methylation pattern can occur at the similar frequency in both cancer and neighboring tissues, indicating this change as an early stage event; Otherwise, the altered methylation pattern is the late.

that the pathologically defined neighboring non-cancerous tissues represent the cells residing at the early stage of carcinogenesis (Fig. 2A). Assuming that the genes significantly more frequent methylated in tumor $(\mathrm{C})$ in comparison with the neighboring tissues $(\mathrm{N})$ as the late phase targets and the otherwise as the early phase one, 27 genes were assigned in the late phase and 20 in the early phase (Fig. 2B, unpublished observation). The current efforts at the larger scale involving over 500 patients and 500 tumor associated are under way for a DNA methylation based classification and staging system of liver cancer.

\section{Future perspectives}

The recent surges in interest in DNA methylation and other epigenetic phenomena have best been demonstrated by the ongoing international efforts to decipher both the components and the mechanisms of the epigenetic layer of heredity on a gigantic scale: the epigenome project (initiated in 1998, http:// www.epigenome.org), the Epigenetic Plasticity of the Genome project (initiated in 2004, http://www.epigenome-noe.net), and the Encyclopedia of DNA Elements project (ENCODE, initiated in 2003, http://www.genome.gov/10005107). It is fully anticipated that there will be a rapid improvement in technological capability and in the gaining of new insights into the role and mechanisms of epigenetic phenomena in both development and disease (including cancer) in the near future. The concordant epigenetic behavior of multiple genes up to the whole-genome level will be dealt with in a highly precise manner prediction, with considerable ease, and at ease in the near future.
Acknowledgments This work was supported by grants to Jingde Zhu from the Shanghai Science Foundation (04DZ14006 and 05DZ19318), the National Science Foundation (30570850, 10574134), the National Research Program for Basic Research of China (2004CB518804), the National Research Program for High Technology (2006AA02Z197, 2006AA02Z320) and the European $6^{\text {th }}$ program (LSHB-CT-2005-019067). Thanks also go to Professor Jianren Gu for his constructive comments on this manuscript.

\section{References}

Baylin, S. B. and Ohm, J. E. (2006) Epigenetic gene silencing in cancer-a mechanism for early oncogenic pathway addiction? Nat. Rev. Cancer 6, 107-116.

Belinsky, S. A. (2004) Gene-promoter hypermethylation as a biomarker in lung cancer. Nat. Rev. Cancer 4, 707-717.

Belinsky, S. A., Nikula, K. J., Palmisano, W. A., Michels, R., Saccomanno, G.., Gabrielson, E., Baylin, S. B. and Herman, J. G. (1998) Aberrant methylation of p16(INK4a) is an early event in lung cancer and a potential biomarker for early diagnosis. Proc. Nat. Acad. Sci. USA 95, 11891-11896.

Bird, A. (1992) The essentials of DNA methylation. Cell 70, 5-8.

Bird, A. P. (1986) CpG-rich islands and the function of DNA methylation. Nature 321, 209-213.

Chen, J. X., Zheng, Y., West, M. and Tang, M. S. (1998) Carcinogens preferentially bind at methylated $\mathrm{CpG}$ in the p53 mutational hot spots. Cancer Res. 58, 2070-2075.

Cui, H., Cruz-Correa, M., Giardiello, F. M., Hutcheon, D. F., Kafonek, D. R., Brandenburg, S., Wu, Y., He, X., Powe, N. R. and Feinberg, A. P. (2003) Loss of IGF2 imprinting: a potential marker of colorectal cancer risk. Sci. 299, 1753-1755.

Ding, S., Gong, B. D., Yu, J., Gu, J., Zhang, H. Y., Shang, Z. B., Fei, Q., Wang, P. and Zhu, J. D. (2004) Methylation profile of the promoter $\mathrm{CpG}$ islands of 14 "drug-resistance" genes in hepatocellular carcinoma. World J. Gastroenterol. 10, 34333440 .

Eads, C. A., Danenberg, K. D., Kawakami, K., Saltz, L. B., Blake, C., Shibata, D., Danenberg, P. V. and Laird, P. W. (2000) MethyLight: a high-throughput assay to measure DNA methylation. Nucleic Acid Res. 28, 32.

Eden, A., Gaudet, F., Waghmare, A. and Jaenisch, R. (2003) Chromosomal instability and tumors promoted by DNA hypomethylation. Science 300, 455.

Esteller, M. (2000) Epigenetic lesions causing genetic lesions in human cancer: promoter hypermethylation of DNA repair genes. Eur. J .Cancer 36, 2294-2300.

Esteller, M., Fraga, M. F., Guo, M., Garcia-Foncillas, J., Hedenfalk, I., Godwin, A. K., Trojan, J., Vaurs-Barriere, C., Bignon, Y. J., Ramus, S., Benitez, J., Caldes, T., Akiyama, Y., Yuasa, Y., Launonen, V., Canal, M. J., Rodriguez, R., Capella, G., Peinado, M. A., Borg, A., Aaltonen, L. A., Ponder, B. A., Baylin, S. B. and Herman, J. G. (2001) DNA methylation patterns in hereditary human cancers mimic sporadic tumorigenesis. Hum. Mol. Genet. 10, 3001-3007.

Esteller, M., Risques, R. A., Toyota, M., Capella, G., Moreno, V., Peinado, M. A., Baylin, S. B. and Herman, J. G. (2001) Promoter hypermethylation of the DNA repair gene $\mathrm{O}(6)$ - 
methylguanine-DNA methyltransferase is associated with the presence of $\mathrm{G}: \mathrm{C}$ to A:T transition mutations in $\mathrm{p} 53$ in human colorectal tumorigenesis. Cancer Res. 61, 4689-4692.

Gaudet, F., Hodgson, J. G., Eden, A., Jackson-Grusby, L., Dausman, J., Gray, J. W., Leonhardt, H. and Jaenisch, R. (2003) Induction of tumors in mice by genomic hypomethylation. Science 300, 489-492.

Gospodarowicz, M. K., Miller, D., Groome, P. A., Greene, F. L., Logan, P. A. and Sobin, L. H. (2004) The process for continuous improvement of the TNM classification. Cancer 100, 1-5.

Gu, L., Yu, J., Fei, Q., Zhang, H., Xu, H., Gu, J., Zhu, T. and Zhu J. (2006) The promoter CpG island of the anti-apoptotic gene, BCL2 is hypermethylated in urine sediments of $28.3 \%$ bladder cancer patients. Tumor(China) 26, 497-601.

Hanahan, D. and Weinberg, R. A. (2000) The hallmarks of cancer. Cell 100, 57-70.

Herman, J. G., Graff, J. R., Myohanen, S., Nelkin, B. D. and Baylin, S. B. (1996) Methylation-specific PCR: a novel PCR assay for methylation status of $\mathrm{CpG}$ islands. Proc. Natl. Acad. Sci. USA 93, 9821-9826.

Holm, T. M., Jackson-Grusby, L., Brambrink, T., Yamada, Y., Rideout, W. M., 3rd and Jaenisch, R. (2005) Global loss of imprinting leads to widespread tumorigenesis in adult mice. Cancer Cell 8, 275-285.

Huang, J., Zhang, X., Zhang, M., Zhu, J., Zhang, Y., Lin, Y., Wang, K., Qi, X., Zhang, Q., Liu, G., Yu, J., Cui, Y., Yang, P., Wang, Z. and Han, Z. (2006) Recurrence of DLK1 as an imprimted gene could contribute to human hepatcocellular carcinoma. Carcinogenesis in press.

Jaenisch, R. and Bird, A. (2003) Epigenetic regulation of gene expression: how the genome integrates intrinsic and environmental signals. Nat. Genet. 33, 245-254.

Janzen, V., Forkert, R., Fleming, H. E., Saito, Y., Waring, M. T., Dombkowski, D. M., Cheng, T., DePinho, R. A., Sharpless, N. E. and Scadden, D. T. (2006) Stem-cell ageing modified by the cyclin-dependent kinase inhibitor p16INK4a. Nature 443, 421426.

Jelinic, P. and Shaw, P. (2007) Loss of imprinting and cancer. $J$. Pathol. 211, 261-268.

Jubb, A. M., Quirke, P. and Oates, A. J. (2003) DNA methylation, a biomarker for colorectal cancer: implications for screening and pathological utility. Ann. N. Y. Acad. Sci. 983, 251-267.

Kinzler, K. W. and Vogelstein, B. (1996) Lessons from hereditary colorectal cancer. Cell 87, 159-170.

Kolble, K., Ullrich, O. M., Pidde, H., Barthel, B., Diermann, J., Rudolph, B., Dietel, M., Schlag, P. M. and Scherneck, S. (1999) Microsatellite alterations in serum DNA of patients with colorectal cancer. Lab. Invest. 79, 1145-1150.

Li, J., Fei Q., Yu, J., Zhang, H., Wang, P. and Zhu, J. (2004) The methylation state of the promoter $\mathrm{CpG}$ islands of seven metastasis-associated genes and their expression state in six cell lines of liver origins. Chin. J. Cancer 23, 1-7.

Macdonald, J. S. (1999) Carcinoembryonic antigen screening: pros and cons. Semin. oncology 26, 556-560.

Palmisano, W. A., Divine, K. K., Saccomanno, G., Gilliland, F. D., Baylin, S. B., Herman, J. G. and Belinsky, S. A. (2000) Predicting lung cancer by detecting aberrant promoter methylation in sputum. Cancer Res. 60, 5954-5958.

Radtke, F. and Clevers, H. (2005) Self-renewal and cancer of the gut: two sides of a coin. Sci. 307, 1904-1909.

Rakyan, V. K., Hildmann, T., Novik, K. L., Lewin, J., Tost, J., Cox, A. V., Andrews, T. D., Howe, K. L., Otto, T., Olek, A., Fischer, J., Gut, I. G., Berlin, K. and Beck, S. (2004) DNA methylation profiling of the human major histocompatibility complex: a pilot study for the human epigenome project. PLos. Biol 2, e405.

Reynolds, P. A., Sigaroudinia, M., Zardo, G., Wilson, M. B., Benton, G. M., Miller, C. J., Hong, C., Fridlyand, J., Costello, J. F. and Tlsty, T. D. (2006) Tumor suppressor p16INK4A regulates polycomb-mediated DNA hypermethylation in human mammary epithelial cells. J. Biol. Chem. 281, 24790-24802.

Suzuki, H., Gabrielson, E., Chen, W., Anbazhagan, R., van Engeland, M., Weijenberg, M. P., Herman, J. G. and Baylin, S. B. (2002) A genomic screen for genes upregulated by demethylation and histone deacetylase inhibition in human colorectal cancer. Nat. Genet. 31, 141-149.

Suzuki, H., Watkins, D. N., Jair, K. W., Schuebel, K. E., Markowitz, S. D., Chen, W. D., Pretlow, T. P., Yang, B., Akiyama, Y., Van Engeland, M., Toyota, M., Tokino, T., Hinoda, Y., Imai, K., Herman, J. G. and Baylin, S. B. (2004) Epigenetic inactivation of SFRP genes allows constitutive WNT signaling in colorectal cancer. Nat. Genet. 36, 417-422.

Ting, A. H., Jair, K. W., Schuebel, K. E. and Baylin, S. B. (2006) Differential requirement for DNA methyltransferase 1 in maintaining human cancer cell gene promoter hypermethylation. Cancer res. 66, 729-735.

Tommasi, S., Denissenko, M. F. and Pfeifer, G. P. (1997) Sunlight induces pyrimidine dimers preferentially at 5-methylcytosine bases. Cancer Res. 57, 4727-4730.

Trosko, J. E. and Upham, B. L. (2005) The emperor wears no clothes in the field of carcinogen risk assessment: ignored concepts in cancer risk assessment. Mutagenesis 20, 81-92.

Tsavellas, G., Patel, H. and Allen-Mersh, T. G. (2001) Detection and clinical significance of occult tumour cells in colorectal cancer. Br. J. Surg. 88, 1307-1320.

Ushijima, T., Nakajima, T. and Maekita, T. (2006) DNA methylation as a marker for the past and future. $J$. Gastroenterol. 41, 401-407.

Xu, X., Yu, J., Zhang, H., Sun, M., Gu, J., Du, X., Sun, M., Shi, D., Wang, P., Yang, Z. and Zhu, J. (2004) Methylation profile of the promoter $\mathrm{CpG}$ islands of thirty one genes that may contribute to carcinogenesis of the colorectal cancer. World $J$. Gastroenterol. 10, 3441-3454.

Xu, X. L., Yu, J., Zhang, H. Y., Sun, M. H., Gu, J., Du, X., Shi, D. R., Wang, P., Yang, Z. H. and Zhu, J. D. (2004) Methylation profile of the promoter $\mathrm{CpG}$ islands of 31 genes that may contribute to colorectal carcinogenesis. World $J$. Gastroenterol. 10, 3441-3454.

Yang, Z., Shang, Z., Yu, J., Cai, Y., Zhang, H., Gu, J., Xu, H. and Zhu, J. (2004) The methylation profiles of the promoter CpG island of nine tumor associated genes correlate with their expression in three lung cancer cell lines. Tumor 11, 216-222.

Yu, J., Ni, M., Xu, J., Zhang, H., Gao, B., Gu, J., Chen, J., Zhang, L., Wu, M., Zhen, S. and Zhu, J. (2002) Methylation profiling of twenty promoter-CpG islands of genes which may contribute to hepatocellular carcinogenesis. B.M.C. Cancer 2, 29.

Yu, J., Zhang, H., Gu, J., Lin, S., Li, J., Lu, W., Wang, Y. and Zhu, J. (2004) Methylation profiles of thirty four promoter- 
$\mathrm{CpG}$ islands and concordant methylation behaviours of sixteen genes that may contribute to carcinogenesis of astrocytoma. B.M.C. Cancer 4, 65.

Yu, J., Zhang, H., Gu, J., Lin, S., Li, J., Lu, W., Wang, Y. and Zhu, J. (2004) Methylation profiling of thirty four promoter$\mathrm{CpG}$ islands and concordant methylation behaviors of sixteen genes that may contribute to carcinogenesis of malignant glioma,. B.M.C Cancer 4, 65.

Yu, J., Zhang, H. Y., Ma, Z. Z., Lu, W., Wang, Y. F. and Zhu, J.
D. (2003) Methylation profiling of twenty four genes and the concordant methylation behaviours of nineteen genes that may contribute to hepatocellular carcinogenesis. Cell Res. 13, 319333.

Zhu, J. (2005) The altered DNA methylation pattern and its implications in liver cancer. Cell Res. 15, 272-280.

Zhu, J. (2006) DNA methylation and hepatocellular carcinoma. $J$. Hepatobiliary Pancreat Surg. 13, 265-273. 